\title{
Crocin induces autophagic apoptosis in hepatocellular carcinoma by inhibiting Akt/mTOR activity
}

This article was published in the following Dove Press journal:

OncoTargets and Therapy

\author{
Chong Yao ${ }^{1,2}$ \\ Bing-Bing Liu $^{3}$ \\ Xiao-Dong Qian² \\ $\mathrm{Li}-\mathrm{Q}$ in $\mathrm{Li}^{4}$ \\ Heng-Bin $\mathrm{CaO}^{2}$ \\ Qiao-Sheng Guo' \\ Gui-Fen Zhou ${ }^{5}$
}

'Institute of Chinese Medicinal Materials, Nanjing Agricultural University, Nanjing 210095, China; 2Pharmaceutical Department, Huzhou Central Hospital, Huzhou 3/3003, China; ${ }^{3}$ Pharmaceutical Department, TCM Hospital of Changxin, Huzhou 313100, China; ${ }^{4}$ Huzhou Key Laboratory of Molecular Medicine, Huzhou Central Hospital, Huzhou 3 I 3000, China; ${ }^{5}$ College of Pharmaceutical Science, Zhejiang Chinese Medical University, Hangzhou 310053, China
Correspondence: Qiao-Sheng Guo Institute of Chinese Medicinal Materials, Nanjing Agricultural University, No I, Weigang, Nanjing, Jiangsu, Xuanwu District 210095, China Tel +862584395980

Email qiaosheng_guo@I63.com

Gui-Fen Zhou

College of Pharmaceutical, Zhejiang Chinese Medical University, No 548, Binwen Road, Hangzhou, Zhejiang, Binjiang District 310053, China Email zyhxsj@I26.com
Background: Autophagy induction is a common mechanism for antitumor chemicals in induction of cancer cell death. However, the role of autophagy in crocin-induced apoptosis is barely studied in hepatocellular carcinoma (HCC).

Materials and methods: The influence of crocin on growth, apoptosis, and autophagy and its mutual relations were analyzed by Cell Counting Kit- 8 assay, flow cytometer, EGFP-LC3 puncta analysis, and Western blot in HCC cells. The activities of Akt/mTOR axis and its roles in autophagy regulation were also detected by Western blot in HCC cells treated with crocin. Finally, the roles of Akt/mTOR axis in crocin-induced autophagic apoptosis were analyzed by Western blot and flow cytometer in HCC cells.

Results: The results showed that crocin can induce growth inhibition in a does- and timedependent pattern by apoptosis. Increased LC3 puncta and upregulated LC3-II accumulation was observed as early as at 6 hours in HepG2 and HCCLM3 cells treated with $3 \mathrm{mg} / \mathrm{mL}$ crocin. Moreover, apoptosis analysis using flow cytometer and cleaved poly (ADP-ribose) polymerase detection revealed that autophagy initiation was prior to apoptosis activation in HCC cells treated with crocin. When autophagy was blocked with 3-methyladenine, crocin-induced apoptosis was inhibited in HCC cells. Furthermore, crocin treatment constrained the activities of key proteins in Akt/mTOR signaling, such as p-Akt (S473), p-mTOR (S2448), and p-p70S6K (T389), suggesting that crocin could induce autophagic apoptosis in HCC cells in an Akt/mTOR-dependent mechanism. Indeed, when autophagy was suppressed by forced expression of Akt, the crocininduced apoptosis was also impaired in HCC cells.

Conclusion: The results suggested that crocin could induce autophagic apoptosis in HCC cells by inhibiting Akt/mTOR activity. This study originally revealed that autophagic apoptosis is a novel cytotoxic function of crocin, which lays the theoretical foundation for clinical application of crocin in HCC.

Keywords: crocin, apoptosis, autophagy, Akt/mTOR, autophagic apoptosis

\section{Introduction}

Hepatocellular carcinoma (HCC) is the sixth most common malignant tumor and the third most frequent cause of cancer-related mortality worldwide. Hepatitis B viral (HBV) and hepatitis $\mathrm{C}$ viral infections, habitual drinking, obesity, and hemochromatosis, etc, are the most risk factors for the development of HCC. Due to the high infection rate of $\mathrm{HBV}$, China has higher incidence compared to Europe and North America. ${ }^{1-3}$ According to the Barcelona Clinic Liver Cancer staging system, the extent of HCC are classified into five progressive stages. The stage of HCC determines the treatments, such as surgical resection, liver transplant, local ablation, transarterial chemoembolization 
(TACE), and sorafenib, to be applied. Although the HCC early stage has the most comfortable efficacy due to surgical resection or liver transplant, it is difficult to distinguish early HCC from other liver diseases. Therefore, most HCC patients proceed to advanced stages at their first attendance, and hence, TACE and sorafenib are the feasible treatments for clinical therapy. However, TACE and sorafenib treatment is usually accompanied by severe adverse events. ${ }^{4,5}$ Hence, it is really necessary and urgent to develop novel therapeutic agents for HCC treatment with more efficacy and fewer side effects.

Natural products, mostly extracted from herbaceous plants, is one of the important original sources for the discovery of anticancer drugs or adjuvant chemotherapy drugs. Saffron, the dried dark-red stigma of Crocus sativus L. is a traditional Chinese medicine that has originally been used to improve blood circulation. Afterward, saffron has been widely applied in the treatment of multiple diseases including cancers for its properties such as antioxidative, antiproliferative, anti-inflammatory, and memory improving. ${ }^{6,7}$ The main components of saffron are crocetin and its glucosidic derivatives such as crocin, picrocrocin, safranal, and flavonoids. ${ }^{8}$ Among these components, crocin has the most effective anticancer activity. Apoptosis induction and the associated proliferation inhibition are the major mechanisms of crocin in suppressing cancer progression. ${ }^{7}$ Studies have demonstrated that crocin could induce apoptosis of multiple types of cancerous cells including colorectal cancer, ${ }^{9,10}$ gastric adenocarcinoma, ${ }^{11}$ head and neck cancer ${ }^{12}$ and HCC. ${ }^{13}$

Apoptosis, autophagy, and necroptosis are three types of programmed cell deaths. ${ }^{14,15}$ Unlike apoptosis and necroptosis, whose final destiny is cell death, autophagy exhibits bidirectional roles in cell destiny determination depending on the intensity and duration of inducers. Autophagy is a conserved eukaryotic cell stress system characterized by an increased generation of autophagic vesicles with incorporation of longlived proteins and damaged organelles, which are ultimately digested in lysomes. ${ }^{16-18}$ Moderate and controlled autophagy can help cell to adapt stress stimuli such as nutrient deficiency and reactive oxygen species accumulation to promote cell survival. However, excessive autophagy, impairing the necessary cellular constituent and processes, will activate apoptosis or necroptosis and result in cell death finally, and this process is usually called autophagic cell death. The studies have demonstrated that autophagic cell death accounts for the mechanisms of many anticancer reagents. ${ }^{19,20}$

Studies have shown that crocin exhibits protective roles in liver diseases. Crocin not only prevents early liver damages of liver cancer ${ }^{21,22}$ but it also directly suppresses cell survival in HCC by targeting different downstream effectors to inhibit proliferation and induce apoptosis. ${ }^{13}$ Considering the important roles of autophagy in cell homeostasis, here, we explored its roles in crocin-associated apoptosis. The results suggest that crocin can initially induce HCC cell autophagy and subsequently activate HCC apoptosis, which means that crocin-induced autophagy promotes HCC apoptosis and cell death.

\section{Materials and methods Cell culture}

Human HCC cell lines HepG2 and HCCLM3 were originally purchased from Cell Bank of Type Culture Collection in Shanghai of China. The cells were cultured with RPMI-1640 (Hyclone, Los Angeles, CA, USA) supplied with 10\% fetal bovine serum at $37^{\circ} \mathrm{C}$ in a humidified $5 \% \mathrm{CO}_{2}$ incubator.

\section{Reagents, plasmids, and antibodies}

Crocin and 3-MA were purchased from Sigma-Aldrich (St Louis, MO, USA). Z-VAD-FMK was purchased from Selleck Chemicals (Huston, TX, USA). Annexin V/propidium iodide (PI) apoptosis detection kit and CCK-8 were obtained from Beyotime Biotechnology (Shanghai, China). EGFP-LC3 plasmid was obtained from Addgene (Cambridge, MA, USA). GV140-Akt and GV140-vector plasmids were constructed by GeneChem (Shanghai, China). Lipofectamine ${ }^{\mathrm{TM}} 2000$ was purchased from Thermo Fisher (Carlsbad, CA, USA). Antibodies against p-Akt (S473), p70S6K, p-p70S6K (T389), mTOR, and p-mTOR (S2448) were purchased from CST (Beverly, MA, USA). Antibodies against SQSTM1/p62 and LC3-II were purchased from Abcam (Cambridge, MA, USA). Antibodies against cleaved-PARP (D214) and $\beta$-actin were purchased from Immunoway (Plano, TX, USA). Anti-Rabbit/Mouse IgG H\&L (horseradish peroxidase [HRP]) and goat Anti-Rabbit IgG H\&L (Alexa Fluor ${ }^{\circledR}$ 594) were purchased from Abcam.

\section{CCK-8 assay}

Cell proliferation was measured by using a CCK-8 kit. Briefly, the cells were plated at $1 \times 10^{3}$ cells/well in 96 -well plates and then grew for corresponding days. Before detection, $10 \mu \mathrm{L}$ per well CCK-8 reagent (Beyotime) was added and incubated for 1 hour. The absorbance at $450 \mathrm{~nm}$ of each well was read by Epoch (Bio-Tek, VT, USA). CCK-8 assay was performed three times in triplicate.

\section{Apoptosis detection by flow cytometer}

Apoptosis detection was carried out according to the manufacturer's instructions. Briefly, cells were digested with 
trypsin without EDTA, washed by PBS, stained with Annexin V-FITC and PI, then analyzed by FACS Calibur flow cytometer (BD Biosciences, San Jose, CA, USA).

\section{Plasmid transfection and EGFP-LC3 puncta quantification}

Cells were plated into 24-well plate the day before transfection. Two hours before transfection, the culture media were replaced into RPMI-1640, and then EGFP-LC3 or GV140Akt plasmids were transfected into cells with Lipofectamine 2000 at $1 \mu \mathrm{g} / \mathrm{mL}$ according to manufacture's instruction. Briefly, Lipofectamine 2000 and plasmid was diluted in RPMI-1640 for 5 minutes, then mixed at the ratio of $2 \mu \mathrm{L}$ Lipofectamine 2000 to $1 \mu \mathrm{g}$ plasmid, and added into culture medium after 20 minutes incubation at room temperature. Six hours later, the culture medium containing plasmid was replaced into complete medium. Twenty-four hours after transfection, crocin was added into the culture medium at $3 \mathrm{mg} / \mathrm{mL}$, and the EGFP-LC3 puncta were observed under invert fluorescence microscope (Leica Microsystems, Wetzlar, Germany). To quantify autophagy, the average number of LC3 dots per cell was counted in more than randomly selected 5 fields with at least 100 cells for each group.

\section{Immunofluorescent staining}

Immunofluorescent staining was performed to detect the expression and puncta distribution of LC3 as described previously. Cells were seeded into $24-w e l l$ plate, then fixed with $4 \%$ paraformaldehyde, permeabilized with $0.1 \%$ Triton 100 , blocked with $5 \%$ bovine serum albumin, incubated with primary antibodies overnight at $4^{\circ} \mathrm{C}$, incubated with secondary antibodies conjugated with Alexa Fluor ${ }^{\circledR}$ 594, and counterstained with 4',6-diamidino-2-phenylindole. Then pictures were acquired by an invert fluorescence microscope (Leica Microsystems).

\section{Western blot}

Total protein was separated from cell lysis and subjected to sodium dodecyl sulfate polyacrylamide gel electrophoresis separation, followed by blotting onto a polyvinylidene difluoride membrane (EMD Millipore, Billerica, MA, USA). Blots were blocked with 5\% nonfat dry milk, incubated with primary antibody overnight at $4{ }^{\circ} \mathrm{C}$, followed by incubation with HRP-conjugated secondary antibody. In the end, the signal was visualized; and the images were obtained by an chemiluminescent image system (Bio-Rad Laboratories Inc., Hercules, CA, USA). $\beta$-Actin was detected simultaneously as a loading control.

\section{Statistical analysis}

All experiments were independently carried out three times. Statistical analyses were performed using SPSS version 18.0 statistical software (SPSS Inc., Chicago, IL, USA). The paired Student's $t$-test was adopted to determine the statistical significance. Two-sided $P<0.05$ was considered statistically significant.

\section{Results \\ Crocin could induce growth arrest and apoptosis in HCC cells}

Studies show that crocin can induce apoptosis and proliferation arrest of tumor cells. ${ }^{7,8}$ The antiproliferative effects of crocin on HCC cells has also been revealed. Indeed, Cell Counting Kit-8 (CCK-8) assay showed that the growth of HepG2 and HCCLM3 cells were restrained by crocin treatment in a time- and dose-dependent pattern (Figure 1A). Moreover, flow cytometer assay demonstrated that $3 \mathrm{mg} / \mathrm{mL}$ crocin induced significant apoptosis of HepG2 and HCCLM3 at 24 hours (Figure 1B). Thus, in accordance with previous reports, crocin could induce growth arrest and apoptosis in HCC cells.

\section{Blocking apoptosis can impair the growth inhibition effect of crocin on HCC cells}

Since crocin could induce growth arrest and apoptosis of HCC cells, we further explored the relationship between crocin-induced growth arrest and apoptosis. Here, Z-VADFMK, one of the potent apoptosis inhibitors, was used to reveal the role of apoptosis in crocin-induced growth suppression. As shown in Figure 2A, the CCK-8 assay indicated that the suppressive effect of $3 \mathrm{mg} / \mathrm{mL}$ crocin on HCC cells growth was significantly alleviated when $30 \mu \mathrm{M}$ Z-VAD-FMK were added simultaneously. Accordingly, flow cytometer and Western blot assay showed that the crocinassociated apoptotic rates and cleaved poly (ADP-ribose) polymerase (PARP) level were decreased by Z-VAD-FMK treatment (Figure 2B). These results indicate that apoptosis interference can impair the growth inhibition effect of crocin on HCC cells.

\section{Crocin can induce autophagy in HCC cells}

Although apoptosis induction may serve as the main reason for crocin-induced proliferation arrest, the underlying mechanisms for apoptosis were obscure. The important roles of autophagy in apoptosis were revealed by multiple studies. Thus, to uncover whether crocin can induce autophagy in HCC cells, some experiments were performed to explore 
A

HepG2
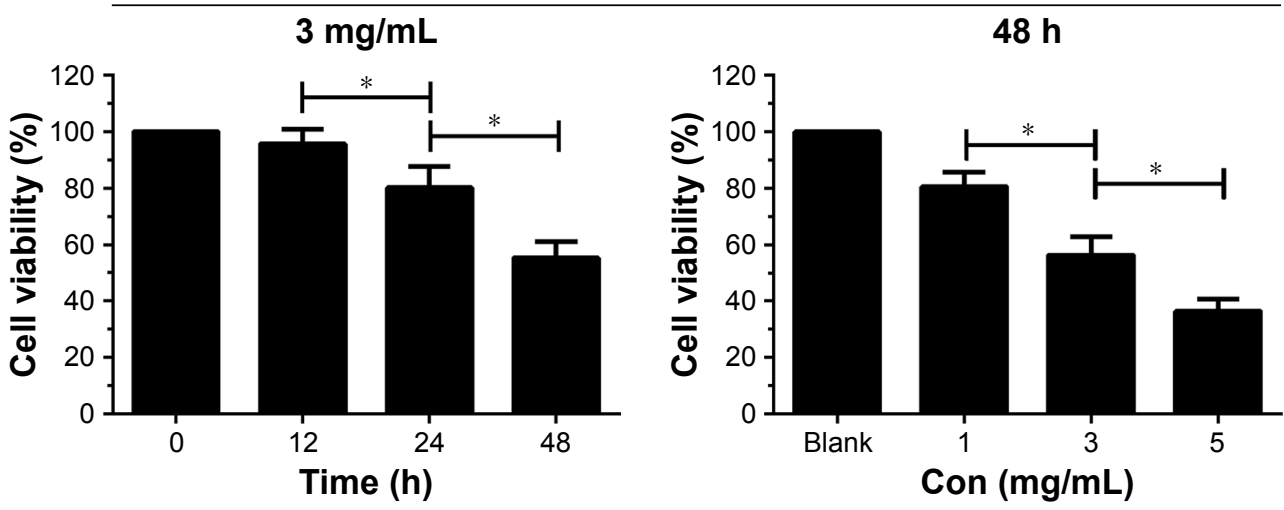

HCCLM3
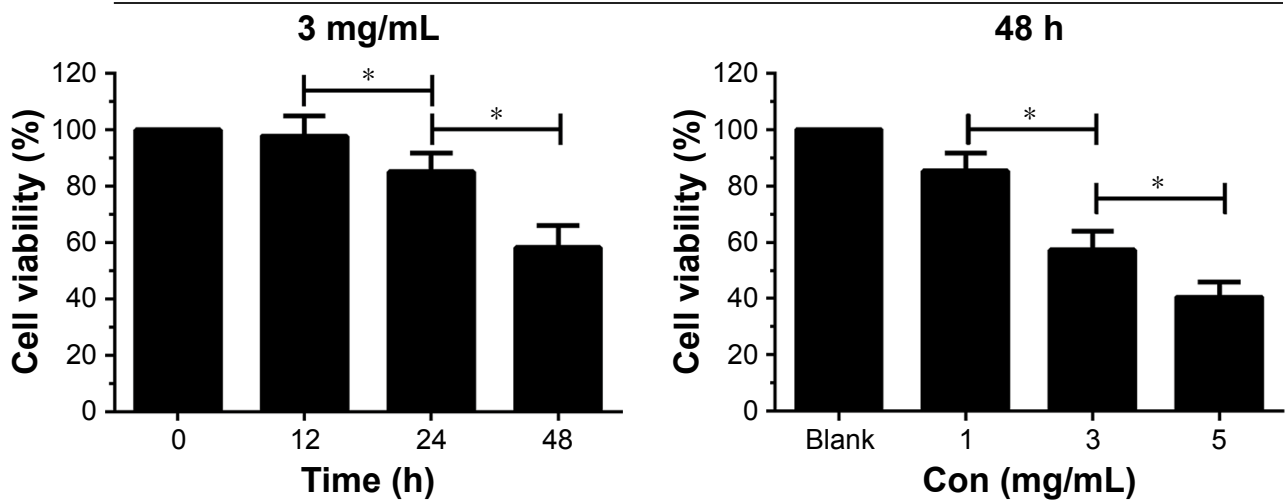

B

HepG2
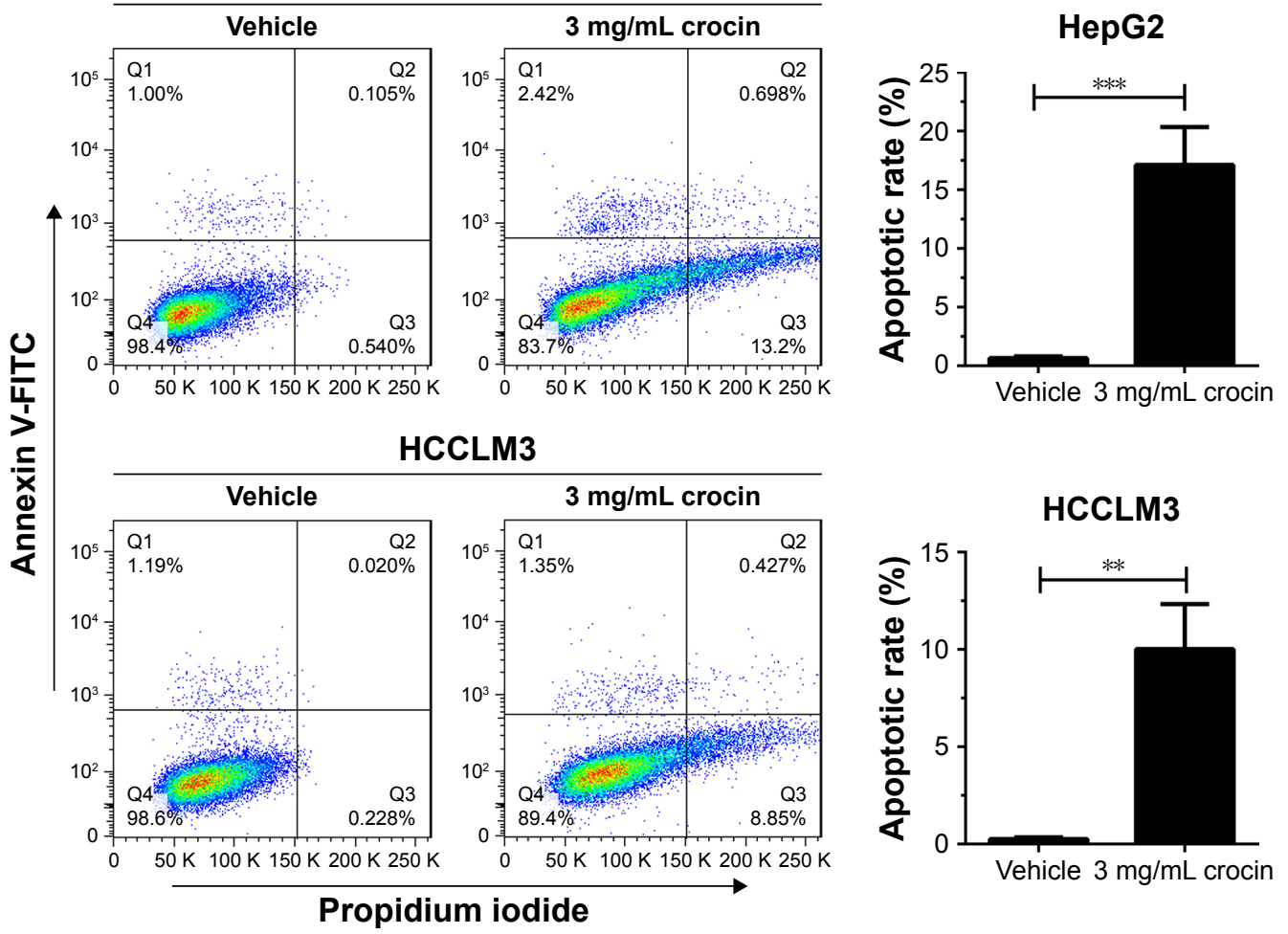

Figure I Crocin inhibited growth and induced apoptosis of HepG2 and HCCLM3 cells.

Notes: (A) CCK-8 assay was used to analyze the inhibitory effect of crocin on HepG2 and HCCLM3 cells. Crocin showed significant inhibitory effect on proliferation of HepG2 and HCCLM3 in the time- and dose-dependent pattern. (B) Flow cytometer was used to analyze the apoptosis of HepG2 and HCCLM3 cells treated by crocin. Crocin of $3 \mathrm{mg} / \mathrm{mL}$ induced significant apoptosis of HepG2 and HCCLM3 cells at 24 hours. $* P<0.5$, $* * P<0.01$, and $* * * P<0.001$.

Abbreviations: CCK-8, Cell Counting Kit-8; h, hours. 
A
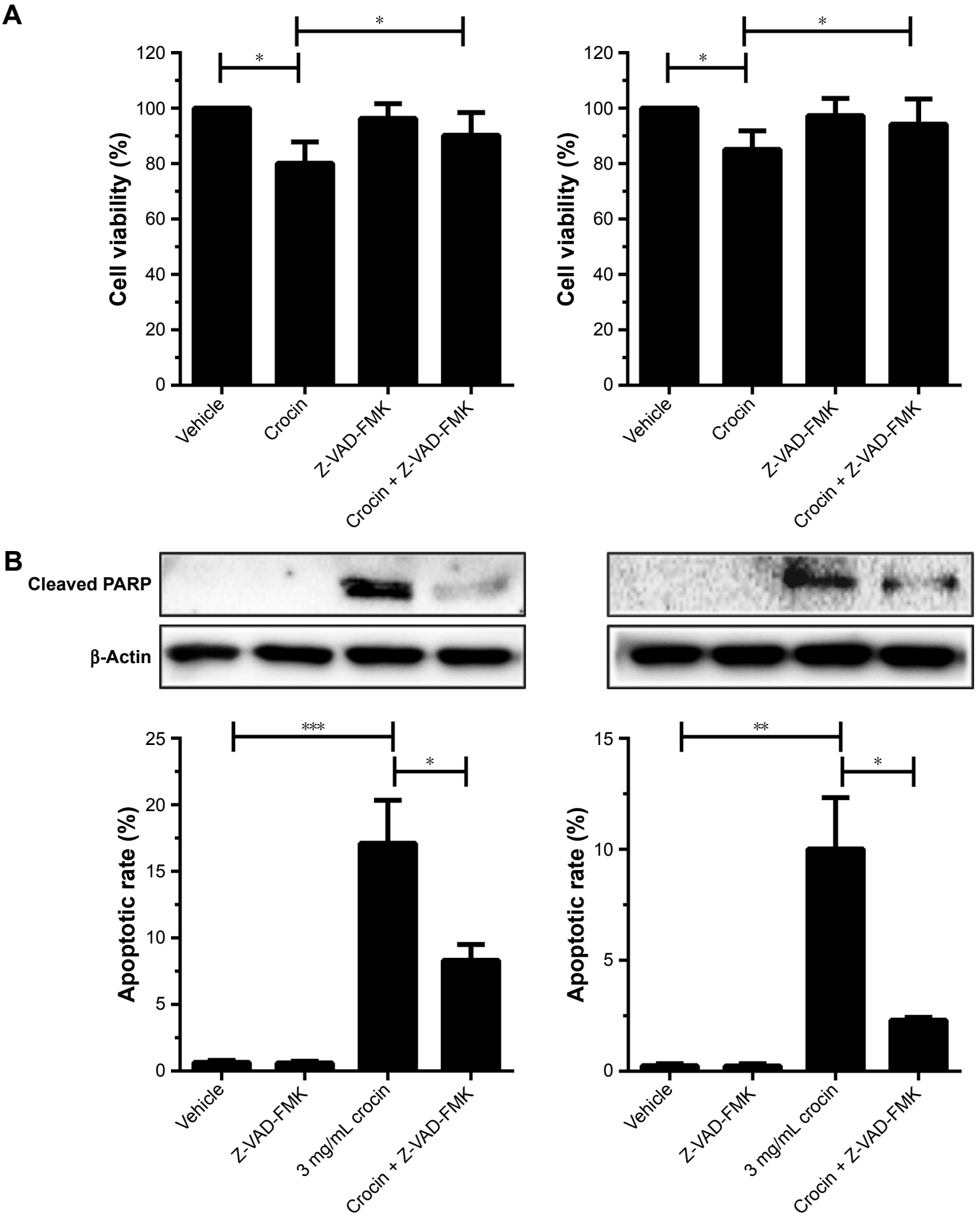

Figure 2 Blockage of apoptosis countered the crocin-induced growth inhibition for HepG2 and HCCLM3 cells.

Notes: $(\mathbf{A})$ The growth of HepG2 and HCCLM3 cells treated by crocin or/and Z-VAD-FMK were analyzed by CCK-8 assay. Z-VAD-FMK of $30 \mu M$ can antagonize the inhibitory effects of crocin on proliferation of HepG2 and HCCLM3 cells. (B) The apoptotic status of HepG2 and HCCLM3 cells treated by crocin or/and Z-VAD-FMK were analyzed by cleaved PARP detection and flow cytometer assay. Z-VAD-FMK of $30 \mu \mathrm{M}$ can antagonize the apoptosis induced by crocin in HepG2 and HCCLM3 cells. $* P<0.05$, **P $<0.01$, and $* * * P<0.001$

Abbreviations: CCK-8, Cell Counting Kit-8; PARP, poly (ADP-ribose) polymerase.

this question. First, EGFP-LC3 plasmid was transfected into HepG2 and HCCLM3 cells, and then autophagy was monitored by EGFP-LC3 puncta. After addition of $3 \mathrm{mg} / \mathrm{mL}$ crocin, EGFP-LC3 puncta were significantly increased as early as at
6 hours, which became stronger at 12 hours in both HepG2 and HCCLM3 cells (Figure 3A). The increased EGFP-LC3 puncta suggested that crocin can induce HCC cells autophagy. Meanwhile, immunofluorescence (IF) was also performed to 

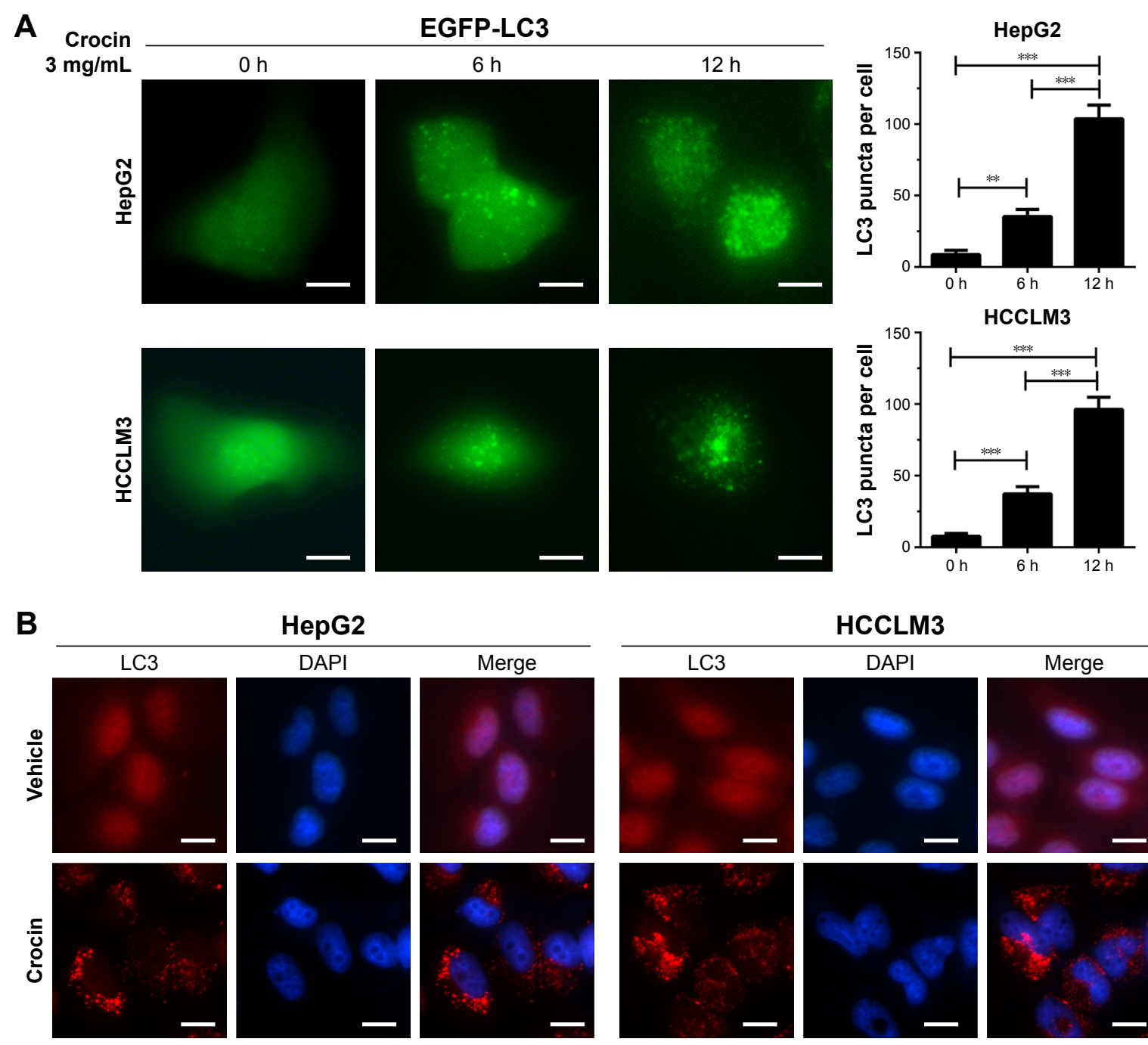

HepG2
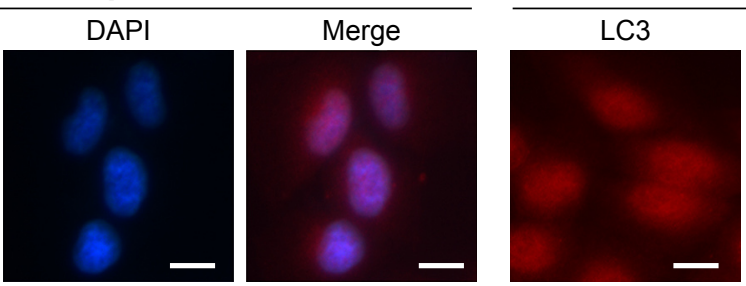

HCCLM3
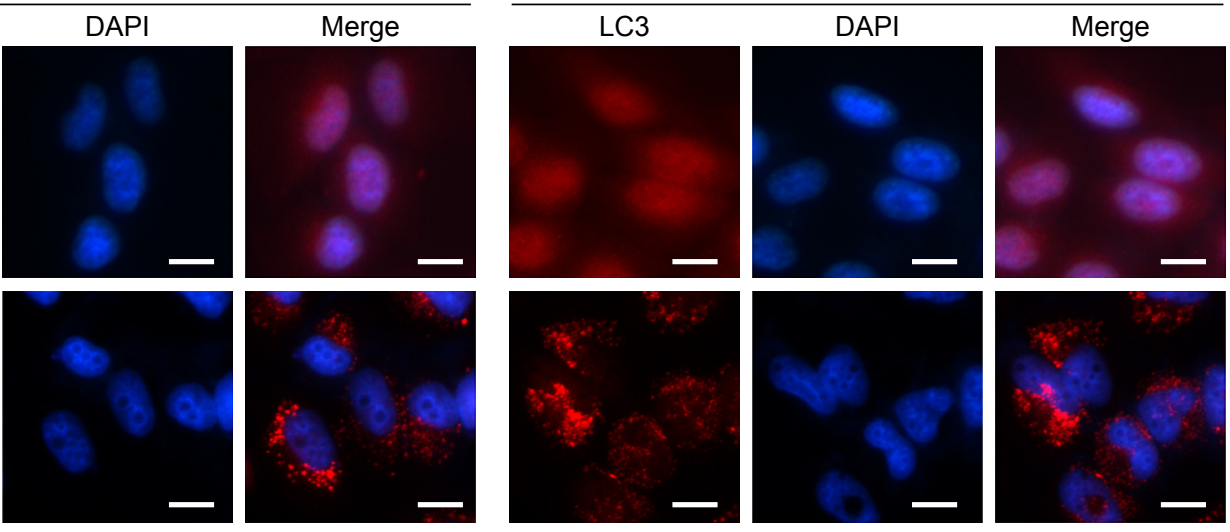

C

HepG2
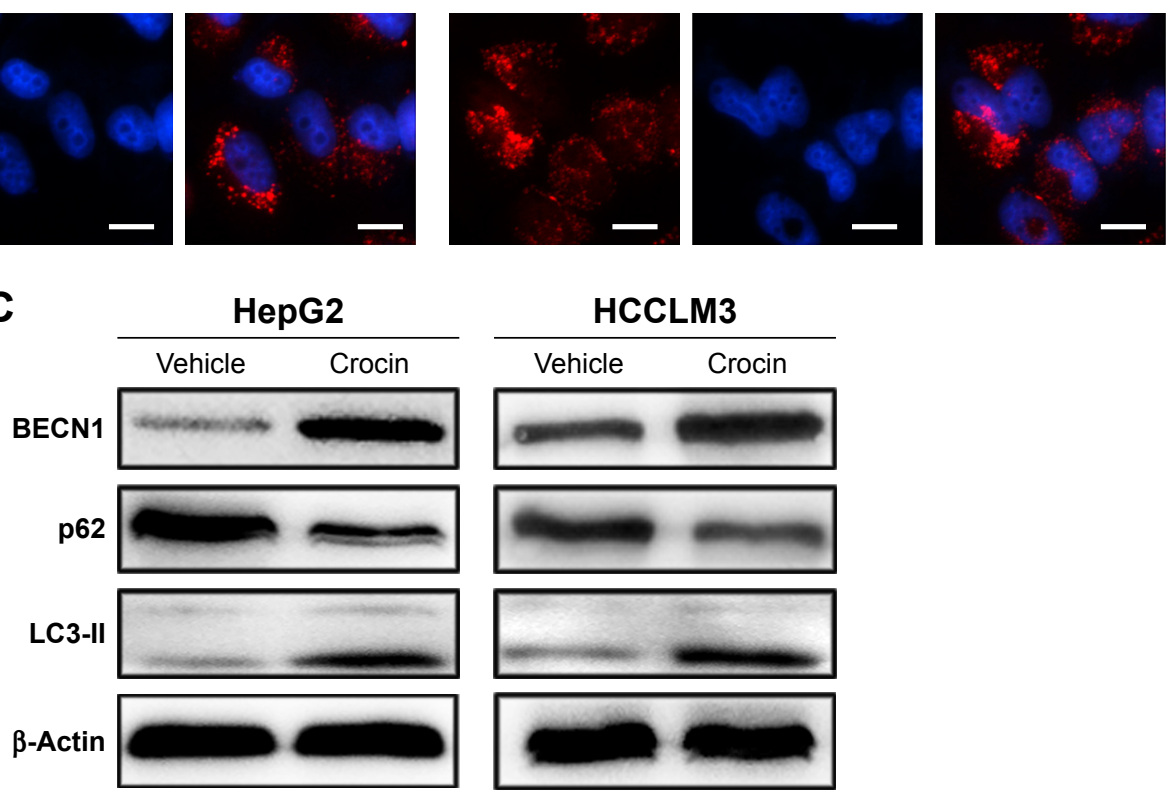

Figure 3 Detecting autophagy in HepG2 and HCCLM3 cells treated by crocin.

Notes: (A) Increased EGFP-LC3 puncta were observed as early as at $6 \mathrm{~h}$ and became stronger at $12 \mathrm{~h}$ in HepG2 and HCCLM3 cells treated with $3 \mathrm{mg} / \mathrm{mL} \mathrm{crocin}$. (B) Representative LC3 puncta were also observed in HepG2 and HCCLM3 cells treated with $3 \mathrm{mg} / \mathrm{mL}$ crocin at 0,6, and I2 h in IF analysis. Magnification: 400×. (C) Western blot analysis showed upregulated BECNI and LC3-II acculumation and downregulated p62 in HepG2 and HCCLM3 cells treated by 3 mg/mL crocin. Magnification: 400. $* * P<0.01$ and $* * * P<0.001$. Scale bars $=20 \mu \mathrm{m}$.

Abbreviation: h, hours.

detect the LC3 puncta formation, and obvious red LC3 puncta were also observed in HepG2 and HCCLM3 cells treated with crocin for 12 hours (Figure 3B). Moreover, upregulated BECN1, decreased p62, and accumulated LC3-II, indicating the activation of autophagic flux, were also detected in HepG2 and HCCLM3 cells treated with crocin by Western blot (Figure 3C). Thus, these results suggest that crocin can induce autophagy in HCC cells. 


\section{Autophagy initiation is earlier than apoptosis activation in HCC cells treated with crocinj}

The studies revealed the dual roles, antiapoptosis and proapoptosis, of autophagy in apoptosis regulation under different stresses. As we proved, crocin could induce autophagy and apoptosis in HCC cells, and it is necessary to explore the relation between crocin-induced autophagy and apoptosis. Thus, the autophagy and apoptosis level were simultaneously monitored by detecting the level of LC3-II accumulation and cleaved PARP production. Obviously, as shown in Figure 4A, Western blot analysis showed that LC3-II was accumulated as early as at 6 hours, while cleaved PARP production was only detected at 12 hours, which indicated that autophagy induction occurs earlier compared to apoptosis initiation in HCC cells treated with crocin. Accordingly, apoptotic rates of HepG2 and HCCLM3 cells were comparative between 0 hour and 6 hours under crocin treatment. Then, significant increment of apoptotic rates was observed at 12 hours in HepG2 and HCCLM3 cells treated with crocin (Figure 4B).
Therefore, above results demonstrate that autophagy initiation occurs earlier compared to apoptosis activation in HepG2 and HCCLM3 cells treated with crocin, which suggests that autophagy may contribute to the apoptosis of HCC cells treated with crocin.

\section{Autophagy inhibition impairs the inhibitory efficiency of crocin on HCC cells}

Moderate autophagy exerts protective roles in cell adaption; however, excessive autophagy, impairing the necessary cellular constituent and processes, will activate programmed cell death. ${ }^{16,17}$ Considering that autophagy initiation occurs before apoptosis activation in HCC cells treated with crocin, autophagy was modified to analyze its role in apoptosis. When autophagy was inhibited by 3-methyladenine (3-MA), an autophagy initiation inhibitor, the apoptosis of HepG2 and HCCLM3 cells was also suppressed, which is reflected by the decreased cleaved PARP level under crocin treatment (Figure 5A). The accordant results were observed in flow cytometry apoptosis analysis, which shows that
A

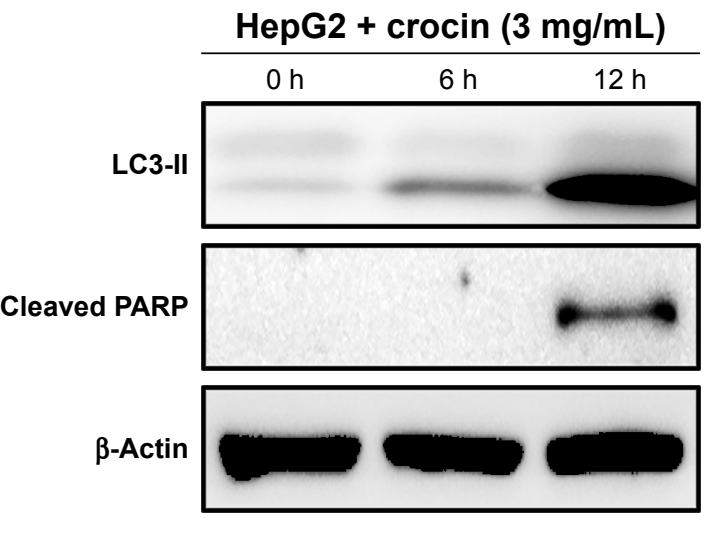

B

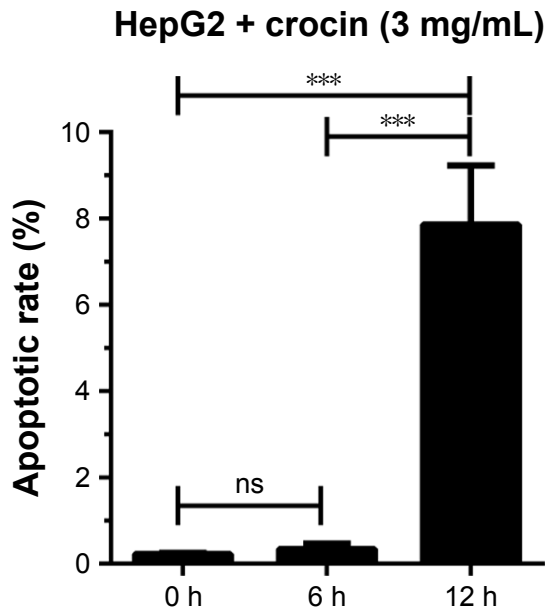

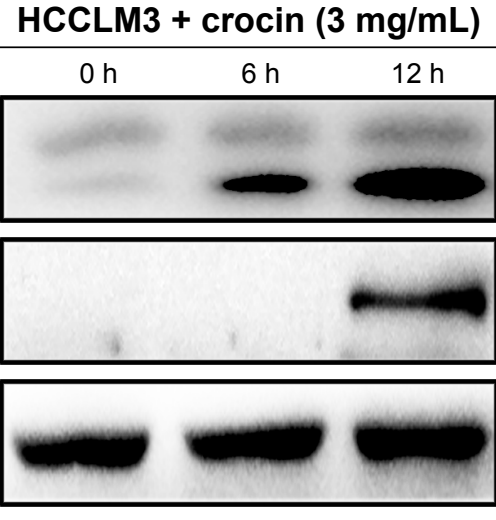

HCCLM3 + $\operatorname{crocin}(3 \mathrm{mg} / \mathrm{mL})$

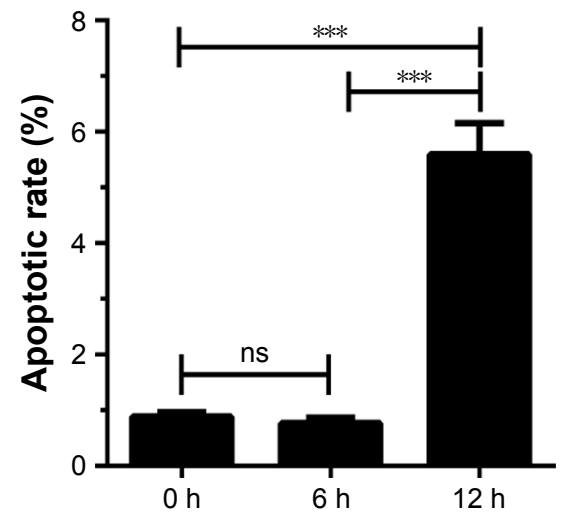

Figure 4 Analyzing the association between autophagy and apoptosis in HepG2 and HCCLM3 cells treated with crocin.

Notes: (A) Western blot analysis showed that LC3-II accumulation was earlier cleaved-PARP production, suggesting that autophagy induction was earlier than apoptosis, (B) which was verified by flow cytometer-mediated apoptosis analysis. $* * * P<0.001$.

Abbreviations: h, hours; ns, no significance; PARP, poly (ADP-ribose) polymerase. 
A

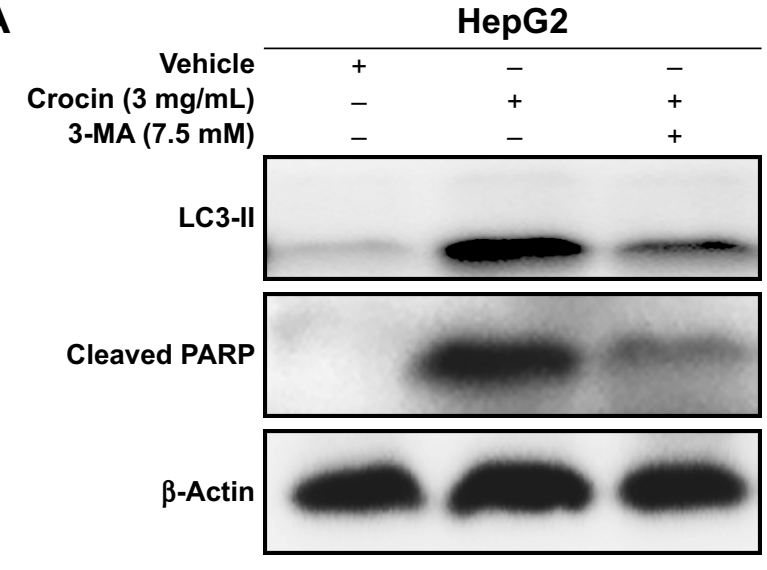

B

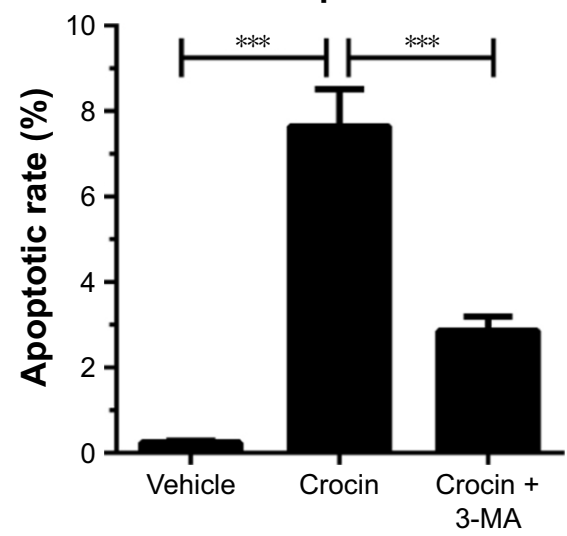

HCCLM3

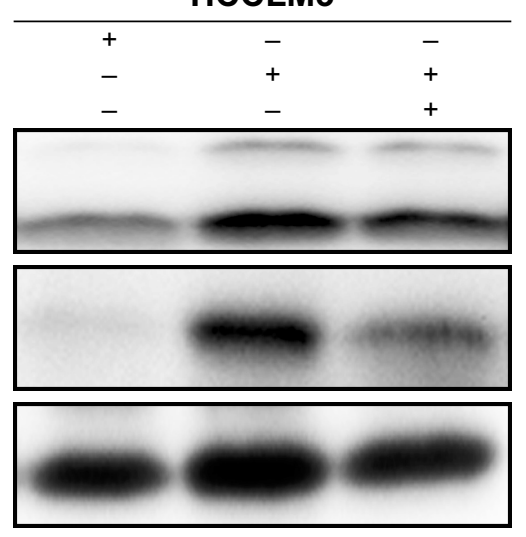

HCCLM3

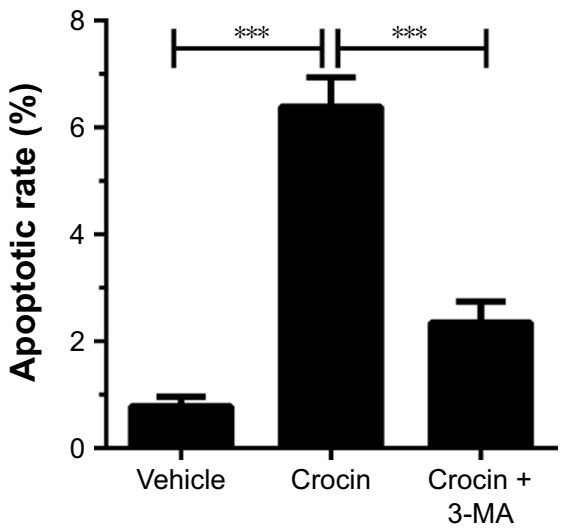

Figure 5 Analyzing the roles of autophagy in apoptosis in HepG2 and HCCLM3 cells treated with crocin.

Notes: (A) Western blot analysis showed that $7.5 \mathrm{mM} 3-\mathrm{MA}$ addition inhibited cleaved PARP production induced by crocin in HepG2 and HCCLM3 cells. (B) Flow cytometer-mediated apoptosis analysis showed that 3-MA addition decreased apoptotic rates induced by crocin in HepG2 and HCCLM3 cells. $* * * P<0.00$ I.

Abbreviations: 3-MA, 3-methyladenine; PARP, poly (ADP-ribose) polymerase.

crocin-associated apoptotic effects were antagonized by 3-MA in HepG2 and HCCLM3 cells (Figure 5B). Taken together, these data suggest that autophagy induction served as an early step for apoptosis induction in HCC cells treated with crocin and inhibits autophagy could suppress crocininduced apoptosis in HCC cells.

\section{Crocin induces HCC cells autophagy in an Akt/mTOR-dependent pattern}

Exogenous or endogenous stresses could activate autophagy by targeting different signal regulators. Among these, Akt/ mTOR axis is extensively studied, which plays an important role in autophagy regulation. ${ }^{18,23}$ Several herbal compounds, such as neferine, ${ }^{24}$ hederagenin, ${ }^{25}$ could activate autophagy by inhibiting mTOR. So, the activity of Akt/mTOR signaling axis of HCC cells treated with crocin were analyzed by Western blot. As shown in Figure 6A, the protein levels of p-Akt (S473), p-mTOR (S448), and p-p70S6K (T389) were downregulated under crocin treatment indicating that crocin may activate autophagy in HepG2 and HCCLM3 cells by inhibiting Akt/mTOR axis. Subsequently, Akt plasmids were introduced into HepG2 and HCCLM3 cells, and then the expression levels of autophagy marker were detected under crocin treatment. As shown in Figure 6B, Akt overexpression almost reactivated the activity of $\mathrm{Akt} / \mathrm{mTOR}$ and suppressed autophagy in HepG2 and HCCLM3 cells treated with crocin. Thus, these results demonstrate that crocin could induce HCC cell autophagy in an Akt/mTOR-dependent pattern.

\section{Reactivation of $A k t / m T O R$ axis can antagonize crocin-induced autophagic apoptosis in HCC cells}

Considering that Akt/mTOR axis is the target for crocininduced autophagy in HCC cells, we further explore its functions in crocin-induced autophagic apoptosis. As shown in Figure 7A, reactivation of Akt/mTOR signaling by forced expression of Akt antagonized the upregulated LC3-II and cleaved PARP level induced by crocin in HepG2 


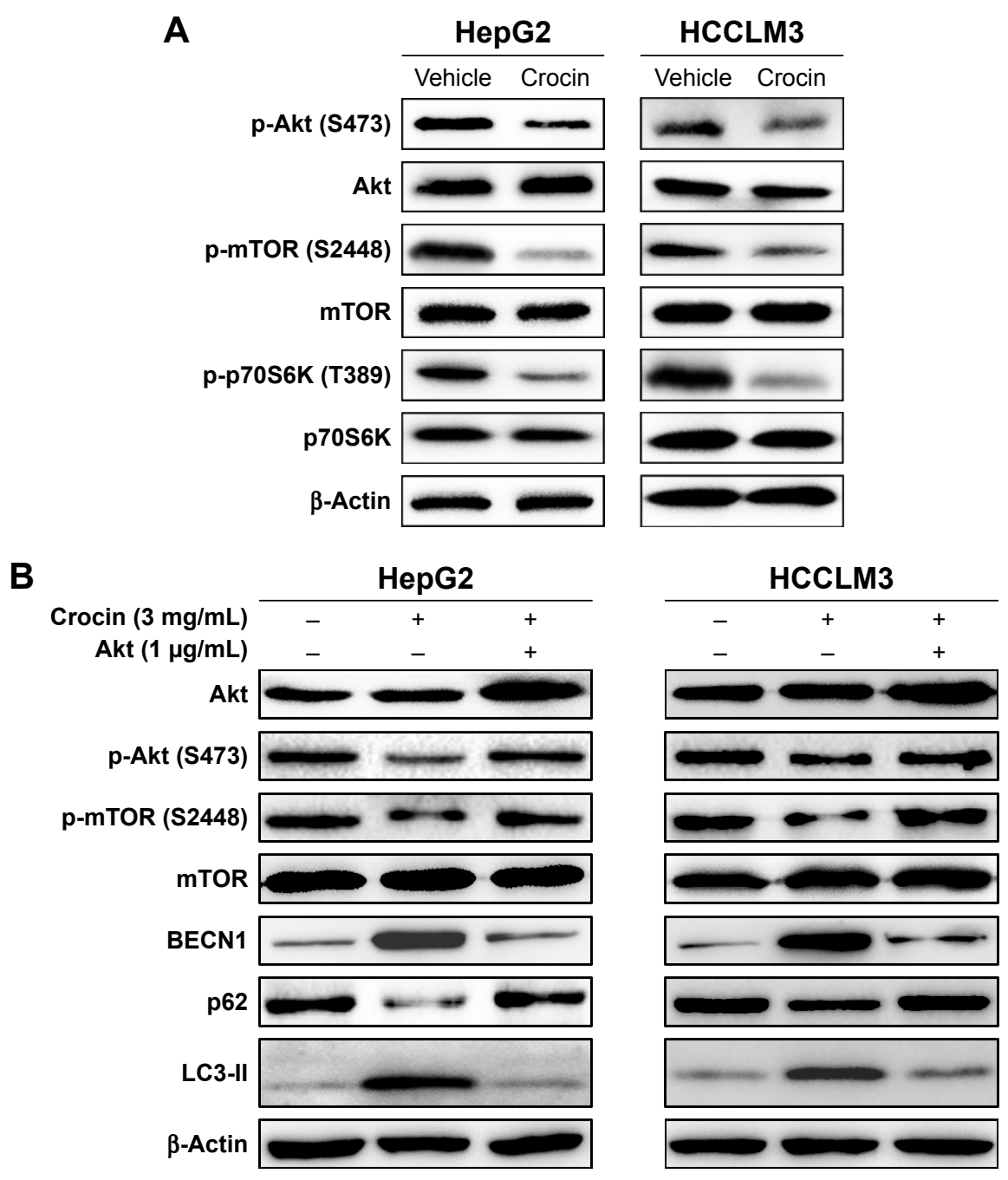

Figure 6 Analyzing the role of Akt/mTOR signaling in crocin-induced autophagy of HepG2 and HCCLM3 cells.

Notes: (A) Western blot analysis showed that the protein levels of Akt/mTOR axis such as p-Akt (S473), p-mTOR (S2448), and p-p70S6K (T389) were suppressed in HepG2 and HCCLM3 cells treated with $3 \mathrm{mg} / \mathrm{mL}$ crocin. (B) Akt overexpression can counteract the effects of crocin by reactivating Akt/mTOR axis activity and subsequently reinhibiting autophagy induction reflected by increased level of p-Akt (S473), p-mTOR (S2448), p62, and decreased BECNI.

and HCCLM3 cells. Moreover, the consistent results were observed in flow cytometry apoptosis analysis. Akt overexpression reinforced the tolerance of HCC cells to crocin-induced apoptosis reflected by lower apoptotic rates (Figure 7B). Thus, these results demonstrate that crocin induces autophagic apoptosis in HCC cells by inhibiting Akt/mTOR axis.

\section{Discussion}

As a precious herbal medicine, the saffron is widely used in the treatment of multiple diseases including cardiovascular and cerebrovascular diseases, inflammation-associated diseases, autoimmune diseases, and cancers for its antioxidant and anti-inflammatory abilities. ${ }^{6-8}$ Crocin, a main active component of saffron, has been extensively studied for its water solubility property, which has the advantage of being consumed orally. Now, it is commercially available as dissolving tablets. ${ }^{26,27}$ Therefore, the potentials of crocin in cancer prevention and therapy are received more and more attention in both theoretical and clinical researches. The emerging studies have demonstrated that crocin could inhibit cancer cell proliferation and induce cell apoptosis by different mechanisms such as suppressing activities of key enzymes in nucleic acid synthesis, remodifying epigenetic properties, and so on. ${ }^{7}$ As for $\mathrm{HCC}$, crocin has shown protective roles for prevention of early liver lesion in a HCC mouse model..$^{22}$ Moreover, crocin could inhibit HepG2 proliferation and induce apoptosis by suppressing the activity of $h T E R T .^{13}$ In this study, we verify the abilities of apoptosis induction and growth arrest of crocin in HCC cells and further reveal that apoptosis attribute a lot to growth inhibition in HCC cells under crocin treatment. Moreover, we find that crocin could 
A

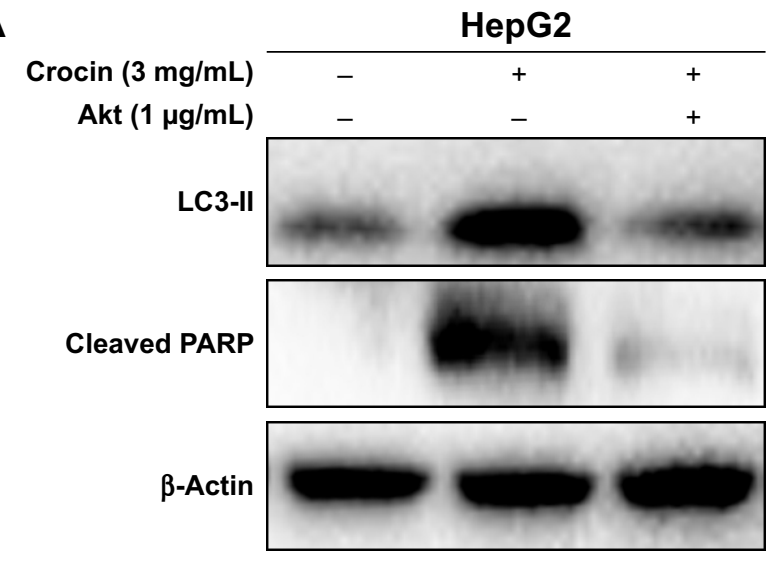

B

HepG2

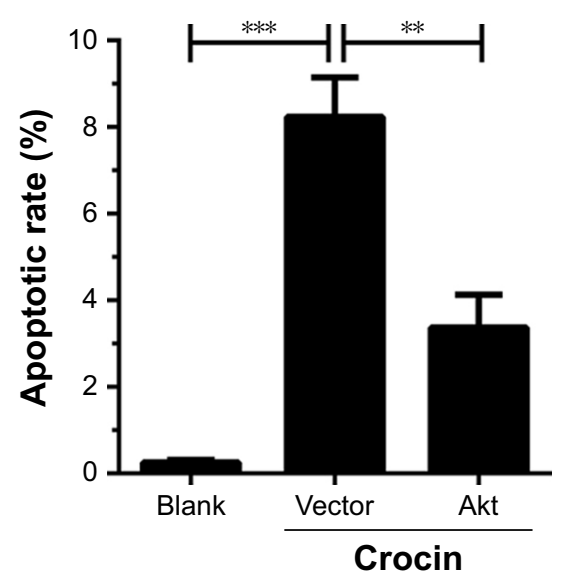

HCCLM3

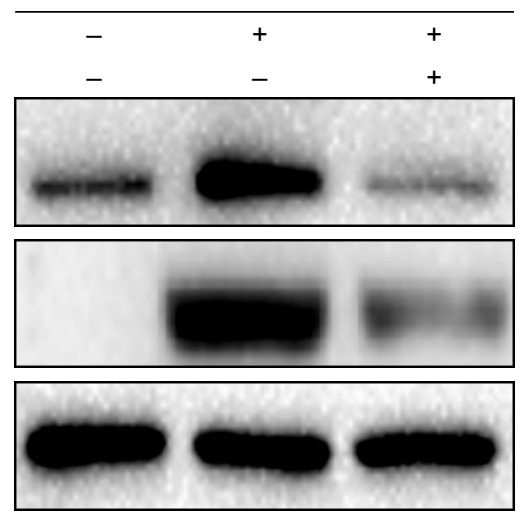

HCCLM3

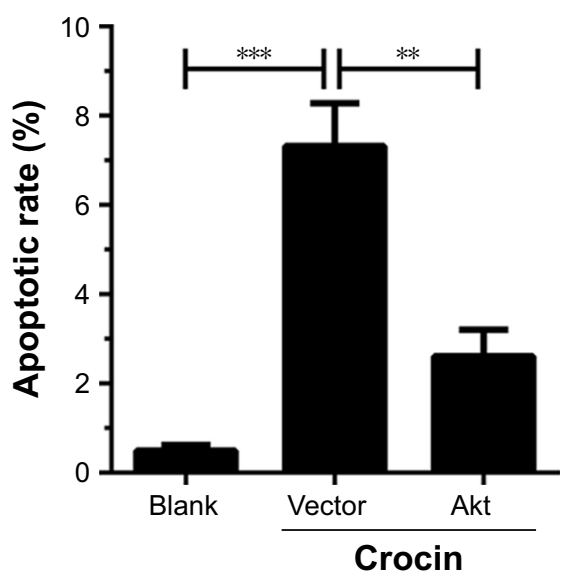

Figure 7 Analyzing the role of Akt/mTOR signaling in crocin-induced autophagic apoptosis of HepG2 and HCCLM3 cells.

Notes: (A) Western blot analysis showed that Akt overexpression inhibited LC3-II accumulation and cleaved PARP production induced by crocin in HepG2 and HCCLM3 cells. (B) Flow cytometer-mediated apoptosis analysis showed that Akt overexpression decreased apoptotic rates induced by crocin in HepG 2 and $\mathrm{HCCLM} 3$ cells. **P $<0.0 \mathrm{I}$ and $* * * P<0.001$.

Abbreviations: mTOR, mechanistic target of rapamycin; PARP, poly (ADP-ribose) polymerase.

activate autophagy in an AKT/mTOR-dependent way, which exerts a positive role in crocin-induced apoptosis. Taken together, our study reveals that crocin can induce autophagic apoptosis in HCC by inhibiting AKT/mTOR activity.

Apoptosis, a common way of cell death, is the main mechanism for antitumor chemical agents that induced growth inhibition. As for crocin, its inhibitory effect on cell growth can be attributed to induction of apoptosis in multiple cancerous cells including cervical cancer, breast cancer, lung cancer, and hematological malignancies. ${ }^{28-32}$ In HCC, both in vivo and in vitro studies have indicated that crocin can inhibit cell proliferation and induce apoptosis, which may be associated with suppression of $h T E R T$. However, the direct relationship between apoptosis and growth inhibition was not explored. Here, we confirm the apoptosis induction and growth inhibition of crocin in HCC cells. Moreover, by using Z-VAD-FMK, we directly uncover that apoptosis induction accounts for the growth inhibition in HCC cells.
Apoptosis, autophagy, and necroptosis, three forms of programmed cell deaths, play vital roles in homeostasis maintenance and adaption to different stresses in independent or cooperative patterns. ${ }^{15}$ Especially, the interplay between apoptosis and autophagy are extensively studied, and several common regulators are identified. Apoptosisassociated proteins such as Bcl-2 family proteins, p53, and caspase family proteins and autophagy-associated proteins such as ATG5, Beclin 1, and mTOR can exert mutual functions in apoptosis and autophagy. ${ }^{33-35}$ As mentioned above, autophagy exhibits dual roles in cell destiny determination, and its relation to apoptosis can be classified into three forms: concomitant occurrence with independent regulation, an early step of apoptosis induction, and apoptosis antagonism. ${ }^{34,35}$ Considering crocin could induce HCC cells apoptosis both in vivo and in vitro, ${ }^{13,22}$ therefore, we wonder whether crocin could induce autophagy, and, if so, what is the role of crocin-induced 
apoptosis? In this study, our results showed that crocin can also induce HCC cell autophagy. Moreover, by dynamic monitoring autophagy and apoptosis, we revealed that autophagy initiation was earlier than apoptosis activation in crocin-treated HepG2 and HCCLM3 cells. Moreover, the apoptosis was suppressed when autophagy was blocked by 3-MA, which suggested that autophagy served as an early step for crocin-induced apoptosis in HCC cells.

Autophagy is an evolutionarily conserved process that sequesters nonessential intracellular components for lysosomal degradation in response to a variety of stress stimuli. According to the pathways to deliver the cargo, autophagy can be subdivided into macroautophagy, microautophagy, and chaperone-mediated autophagy. Usually, macroautophagy is generally referred to as "autophagy." Autophagy initialization is dependent on a key protein complex including Vps34 (Class III PI3K), Beclin-1, and other interacted proteins. ${ }^{16,17,36}$ Altering the activity of core proteins and then changing the core protein-interacted patterns are the common mechanism of upstream signaling regulators. As the most cell signaling axis, Akt/mTOR can negatively regulate autophagy by inhibiting ULK1. mTOR serves as a cellular sensor of nutrient, growth factor availability, energy levels, and stress signals, which is positively regulated by nutrient and growth factors. Under cell stress conditions, such as nutrient deprivation, growth factor shortage, and hypoxia, and so on, Akt/mTOR axis is inhibited to induce autophagy. ${ }^{18,36}$ Consequently, Akt/mTOR is a common target for autophagy inducers. For example, multiple herbal compounds such as tetrandrine, ${ }^{19}$ onjisaponin $\mathrm{B},{ }^{37}$ neferine, ${ }^{24}$ hederagenin, and $\alpha$-hederin ${ }^{25}$ could induce autophagy by inhibiting mTOR activity. Conversely, AMPK, another cellular energy sensor, is negatively regulated by energy level, which promotes the transition from anabolic to catabolic metabolism upon energy stress. This role suggests a positive link between AMPK and autophagy regulation. Upon stimuli, AMPK ca activate autophagy by directly phosphorylating and activating ULK1 or indirectly activating ULK1 with TSC2-mediated mTOR inhibition. In our study, in HepG2 and HCCLM3 cells treated with crocin, the activity of Akt/mTOR axis was suppressed; meanwhile, the activity of AMPK was not fluctuated (data not shown). Thus, Akt/mTOR axis inhibition accounts for the autophagy activation induced by crocin in HCC cells.

In this study, we originally reveal that crocin can induce autophagy of HCC cells by inhibiting Akt/mTOR axis activity and present that autophagy inhibition leads to apoptosis resistance in crocin-treated HCC cells, which reports a new antitumor mechanism of crocin and offer theoretical foundation for the clinical application of crocin.

\section{Acknowledgment}

This study was supported by National Natural Science Foundation of China (81403032 and 31600255) and Zhejiang Provincial Natural Science Foundation of China (LQ15H280001).

\section{Author contributions}

All authors contributed toward data analysis, drafting and critically revising the paper and agree to be accountable for all aspects of the work.

\section{Disclosure}

The authors report no conflicts of interest in this work.

\section{References}

1. Cidon EU. Systemic treatment of hepatocellular carcinoma: past, present and future. World J Hepatol. 2017;9(18):797-807.

2. Song JE, Kim DY. Conventional vs drug-eluting beads transarterial chemoembolization for hepatocellular carcinoma. World J Hepatol. 2017;9(18):808-814.

3. Ma-On C, Sanpavat A, Whongsiri P, et al. Oxidative stress indicated by elevated expression of $\mathrm{Nrf2}$ and 8-OHdG promotes hepatocellular carcinoma progression. Med Oncol. 2017;34(4):57.

4. Ding XX, Zhu QG, Zhang SM, et al. Precision medicine for hepatocellular carcinoma: driver mutations and targeted therapy. Oncotarget. 2017;8(33):55715-55730.

5. Higashi T, Hayashi H, Kitano Y, et al. Statin attenuates cell proliferative ability via TAZ (WWTR1) in hepatocellular carcinoma. Med Oncol. 2016;33(11):123.

6. Moradzadeh M, Sadeghnia HR, Tabarraei A, Sahebkar A. Anti-tumor effects of crocetin and related molecular targets. J Cell Physiol. 2017; 233(3):2170-2182.

7. Hoshyar R, Mollaei H. A comprehensive review on anticancer mechanisms of the main carotenoid of saffron, crocin. J Pharm Pharmacol. 2017;69(11):1419-1427.

8. Patel S, Sarwat M, Khan TH. Mechanism behind the anti-tumour potential of saffron (Crocus sativus L.): the molecular perspective. Crit Rev Oncol Hematol. 2017;115:27-35.

9. Aung HH, Wang CZ, Ni M, et al. Crocin from Crocus sativus possesses significant anti-proliferation effects on human colorectal cancer cells. Exp Oncol. 2007;29(3):175-180.

10. Amin A, Bajbouj K, Koc 'h A, Gandesiri M, Schneider-Stock R. Defective autophagosome formation in p53-null colorectal cancer reinforces crocin-induced apoptosis. Int J Mol Sci. 2015;16(1):1544-1561.

11. Hoshyar R, Bathaie SZ, Sadeghizadeh M. Crocin triggers the apoptosis through increasing the $\mathrm{Bax} / \mathrm{Bcl}-2$ ratio and caspase activation in human gastric adenocarcinoma, AGS, cells. DNA Cell Biol. 2013; 32(2):50-57.

12. Vazifedan V, Mousavi SH, Sargolzaei J, Soleymanifard S, Fani Pakdel A. Study of crocin \& radiotherapy-induced cytotoxicity and apoptosis in the head and neck cancer (HN-5) cell line. Iran J Pharm Res. 2017;16(1): 230-237.

13. Noureini SK, Wink M. Antiproliferative effects of crocin in HepG2 cells by telomerase inhibition and hTERT down-regulation. Asian Pac J Cancer Prev. 2012;13(5):2305-2309.

14. Fitzwalter BE, Thorburn A. Recent insights into cell death and autophagy. FEBS J. 2015;282(22):4279-4288.

15. Su Z, Yang Z, Xu Y, Chen Y, Yu Q. MicroRNAs in apoptosis, autophagy and necroptosis. Oncotarget. 2015;6(11):8474-8490.

16. Liu J, Fan L, Wang H, Sun G. Autophagy, a double-edged sword in anti-angiogenesis therapy. Med Oncol. 2016;33(1):10. 
17. Li YJ, Lei YH, Yao N, et al. Autophagy and multidrug resistance in cancer. Chin J Cancer. 2017;36(1):52.

18. Noda T. Regulation of autophagy through TORC1 and mTORC1. Biomolecules. 2017;7(3):E52.

19. Wong VKW, Zeng W, Chen J, et al. Tetrandrine, an activator of autophagy, induces autophagic cell death via PKC-alpha inhibition and mTOR-dependent mechanisms. Front Pharmacol. 2017;8:351.

20. Wong VK, Li T, Law BY, et al. Saikosaponin-d, a novel SERCA inhibitor, induces autophagic cell death in apoptosis-defective cells. Cell Death Dis. 2013;4:e720.

21. Amin A, Hamza AA, Daoud S, et al. Saffron-based crocin prevents early lesions of liver cancer: in vivo, in vitro and network analyses. Recent Pat Anticancer Drug Discov. 2016;11(1):121-133.

22. Amin A, Hamza AA, Bajbouj K, Ashraf SS, Daoud S. Saffron: a potential candidate for a novel anticancer drug against hepatocellular carcinoma. Hepatology. 2011;54(3):857-867.

23. Saxton RA, Sabatini DM. mTOR signaling in growth, metabolism, and disease. Cell. 2017;168(6):960-976.

24. Wong VK, Wu AG, Wang JR, Liu L, Law BY. Neferine attenuates the protein level and toxicity of mutant huntingtin in PC-12 cells via induction of autophagy. Molecules. 2015;20(3):3496-3514.

25. Wu AG, Zeng W, Wong VK, et al. Hederagenin and alpha-hederin promote degradation of proteins in neurodegenerative diseases and improve motor deficits in MPTP-mice. Pharmacol Res. 2017;115:25-44.

26. Naess SN, Elgsaeter A, Foss BJ, et al. Hydrophilic carotenoids: surface properties and aggregation of crocin as a biosurfactant. Helv Chim Acta. 2006;89(1):45-53.

27. Mohamadpour AH, Ayati Z, Parizadeh MR, Rajbai O, Hosseinzadeh H. Safety evaluation of crocin (a constituent of saffron) tablets in healthy volunteers. Iran J Basic Med Sci. 2013;16(1):39-46.
28. Mollaei H, Safaralizadeh R, Babaei E, Abedini M, Hoshyar R. The anti-proliferative and apoptotic effects of crocin on chemosensitive and chemoresistant cervical cancer cells. Biomed Pharmacother. 2017; 94:307-316.

29. Kim B, Lee K, Park B. Crocin suppresses constitutively active STAT3 through induction of protein tyrosine phosphatase SHP-1. J Cell Biochem. 2017;118(10):3290-3298.

30. Sun Y, Wang Z, Wang L, Wang LZ, Zang C, Sun LR. The effect and mechanisms of proliferative inhibition of crocin on human leukaemia jurkat cells. West Indian Med J. 2015;64(5):473-479.

31. Mostafavinia S, Khorashadizadeh M, Hoshyar R. Antiproliferative and proapoptotic effects of crocin combined with hyperthermia on human breast cancer cells. DNA Cell Biol. 2016;35(7):340-347.

32. Chen S, Zhao S, Wang X, et al. Crocin inhibits cell proliferation and enhances cisplatin and pemetrexed chemosensitivity in lung cancer cells. Transl Lung Cancer Res. 2015;4(6):775-783.

33. Tsapras P, Nezis IP. Caspase involvement in autophagy. Cell Death Differ. 2017;24(8):1369-1379.

34. Eisenberg-Lerner A, Bialik S, Simon HU, Kimchi A. Life and death partners: apoptosis, autophagy and the cross-talk between them. Cell Death Differ. 2009;16(7):966-975.

35. Lockshin RA, Zakeri Z. Apoptosis, autophagy, and more. Int J Biochem Cell Biol. 2004;36(12):2405-2419.

36. Papinski D, Kraft C. Regulation of autophagy by signaling through the Atg1/ULK1 complex. J Mol Biol. 2016;428(9 pt A):1725-1741.

37. Wu AG, Wong VK, Xu SW, et al. Onjisaponin B derived from Radix Polygalae enhances autophagy and accelerates the degradation of mutant alpha-synuclein and huntingtin in PC-12 cells. Int J Mol Sci. 2013; 14(11):22618-22641.
OncoTargets and Therapy

\section{Publish your work in this journal}

OncoTargets and Therapy is an international, peer-reviewed, open access journal focusing on the pathological basis of all cancers, potential targets for therapy and treatment protocols employed to improve the management of cancer patients. The journal also focuses on the impact of management programs and new therapeutic agents and protocols on

\section{Dovepress}

patient perspectives such as quality of life, adherence and satisfaction The manuscript management system is completely online and includes a very quick and fair peer-review system, which is all easy to use. Visit http://www.dovepress.com/testimonials.php to read real quotes from published authors. 